\section{Impacto de la aplicación del enfoque de autorregulación del aprendizaje sobre los resultados en asignaturas de corte científico en estudiantes de medicina de la Universidad Finis Terrae}

\author{
CAROLINA WILLIAMS ${ }^{\mathrm{a}, \mathrm{d}}$, LUCÍA SANTELICES ${ }^{\mathrm{a}, \mathrm{e}}$, MARIO ÁVILA ${ }^{\mathrm{b}}$, \\ MAURICIO SOTO ${ }^{\mathrm{c}, \mathrm{f}}$, ALBERTO DOUGNAC
}

\section{The impact of a self-directed teaching approach on academic performance of medical students}

Background: Students should be encouraged to become reflexive and develop autonomous, lifelong learning habits. Therefore, teachers should focus on learning strategies which stimulate autonomous learning. Aim: To assess the impact of a self-directed teaching methodology on the academic performance of medical students in cellular biology and biochemistry. Materials and Methods: During 2013, 85 students received a traditional teaching methodology and during 2014, 85 students received a self-directed learning methodology. The grades obtained and the number of failures in the courses of cellular biology and biochemistry were compared in both groups. Results: The percentages of students approved at the end of the courses during 2013 and 2014 were 64 and 96\% respectively $(p<0.01)$. The grades obtained by the 2014 students were also significantly higher than those obtained by 2013 students. Conclusions: This study confirms that academic performance improves with a self-directed teaching approach.

(Rev Med Chile 2017; 145: 595-602)

Key words: Education, Medical; Education, Medical, Undergraduate; Learning; Teaching.

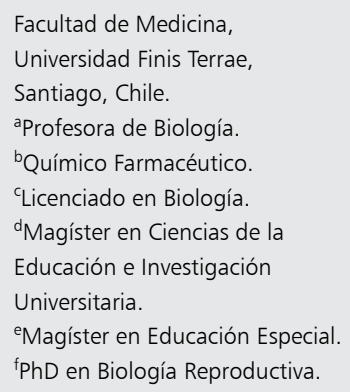

Correspondencia a:

Carolina Williams

Av. Pedro de Valdivia 1509, Providencia, Santiago, Chile. Teléfono: 224207365

cwilliams@uft.cl

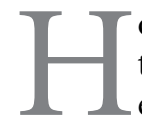
oy en día es primordial que las instituciones de educación superior deban estimular competencias que ayuden a conseguir un aprendizaje continuo y permanente durante toda la vida ${ }^{1,2}$. Para ello es fundamental centrar la mirada en el enfoque metodológico que utiliza el proceso enseñanza aprendizaje. La autorregulación del aprendizaje trabajada como metodología de enseñanza podría responder a la necesidad de contar con estudiantes más autónomos, reflexivos y con capacidad de manejar sus propios procesos.

Los estudios señalan la importancia de este enfoque a nivel cognitivo sobre procesos de planificación, ejecución y reflexión de los estudiantes frente a determinadas tareas ${ }^{2-4}$. La autorregulación del aprendizaje es foco de una gran cantidad de investigaciones ${ }^{2-5}$. Existe consenso en que este proceso puede ser adquirido. Para que ello ocurra, el estudiante debe vivir múltiples experiencias y en diferentes contextos que movilicen componentes personales, conductuales y contextuales ${ }^{4-6}$. Sin embargo, no hay evidencia de cómo se puede dirigir este proceso a través de una metodología de enseñanza docente en carreras de ciencias de la salud ${ }^{6-8}$. 
En el año 2014 se publicó el efecto del enfoque de la autorregulación de aprendizajes sobre la adquisición de conocimientos científicos en cursos de nivelación en ciencias de la Escuela de Medicina de la Universidad Finis Terrae ${ }^{9}$. Sin embargo, surge la necesidad de evaluar si esta metodología, utilizada en los programas de nivelación y en asignaturas científicas de primer año de la carrera de medicina, impacta en el rendimiento académico final de los estudiantes de primer año. Por tanto, los objetivos del estudio apuntan a determinar el impacto de la metodología de la autorregulación del aprendizaje en el rendimiento académico de estudiantes de medicina en cursos de Biología celular y Bioquímica.

\section{Material y Método}

La investigación de corte cuantitativo de tipo cuasi experimental con grupos intactos tiene alcance correlacional. Se trabajó con dos grupos de estudiantes de ingreso a la carrera de medicina (2013 y 2014).

El proceso académico de los estudiantes comienza la primera semana de marzo con: 1) diagnóstico de conocimientos científicos iniciales (pre test); 2) una semana de nivelación; 3) medición de conocimientos científicos finales (post test) y 4) primer semestre académico con asignaturas científicas (Biología celular y Bioquímica). Siguiendo las etapas mencionadas, el grupo 2013 trabajó su proceso académico con una metodología tradicional, mientras el grupo 2014 trabajó con la metodología de autorregulación del aprendizaje. Durante los dos años del estudio participaron los mismos profesores, no hubo cambios de programas de asignaturas, de creditaje, tampoco de instrumentos de evaluación, sólo cambio de metodología para las mismas unidades temáticas.

Durante el segundo semestre de 2013 se capacitó a los docentes para incorporar la autorregulación del aprendizaje en la conducción de sus clases. Se evaluaron las planificaciones de clases incorporando en ellas las fases que involucra la autorregulación según los autores de referencia Pintrich, Zimmerman y Schunk ${ }^{2-4}$.

De los 189 estudiantes matriculados en primer año, 170 participaron del estudio. Los 19 estudiantes que no participaron corresponden a:

1. Ocho estudiantes que estando matriculados no cursaron en forma completa la asignatura ( 3 estudiantes del grupo 2013 y 5 en el grupo 2014).

2. Once estudiantes fueron excluidos por presentar inasistencia durante el desarrollo de una de las dos asignaturas (5 estudiantes del grupo 2013 y 6 en el grupo 2014).

Aplicando los criterios de exclusión, sólo para fines estadísticos, la muestra quedó conformada por 85 estudiantes 2013 y 85 estudiantes 2014 .

El diseño del estudio se observa en la Figura 1.

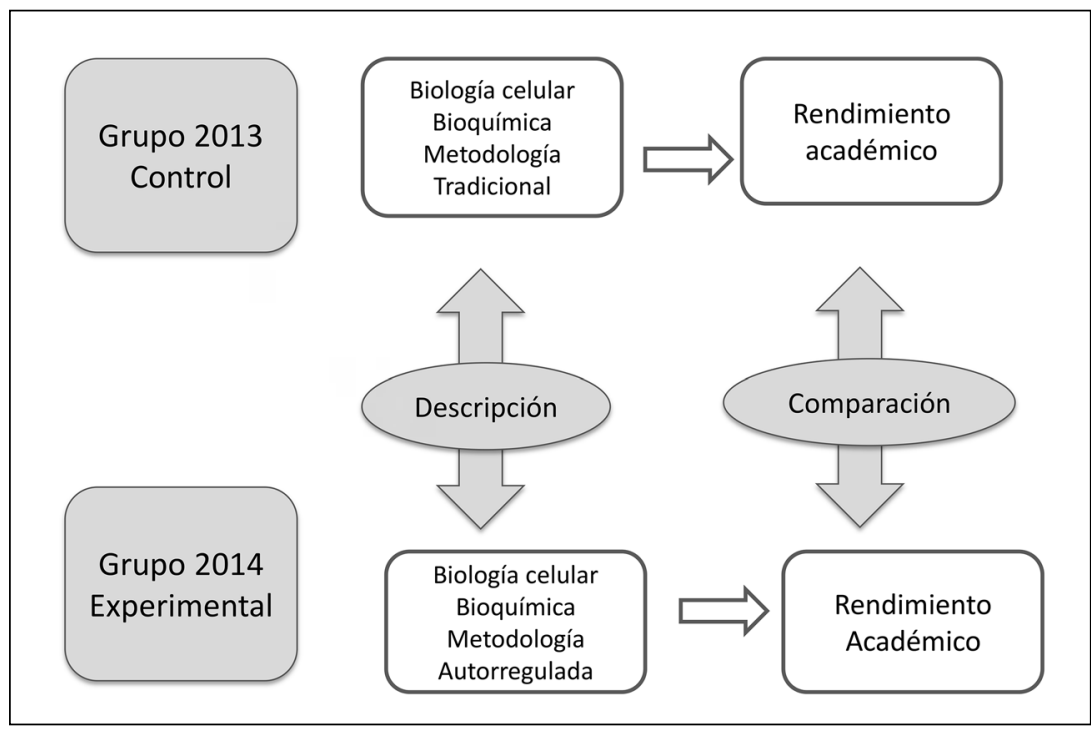

Figura 1. Diseño del estudio. 
Ambos grupos fueron comparables respecto a puntajes de ingreso, sexo, edad, establecimiento educacional; niveles de conocimiento científico iniciales, medido a través de un pre-test estandarizado para la Facultad de Medicina de la Universidad Finis Terrae y validado en el año $2013^{10}$; niveles de comprensión, medido a través de una prueba informal de comunicación efectiva que mide comprensión lectora, vocabulario, capacidad de describir explícitamente lo que se observa (explícito) y capacidad de inferir situaciones que no son evidentes (inferencial) aplicada por un grupo de psicopedagogas pertenecientes a la Vicerrectoría Académica de la universidad desde el año 2010, información que es complementada con la determinación de estilos de aprendizajes y estrategias de estudio de los estudiantes a través del test "Inventory of Learning Processes" del profesor Schmeck ${ }^{11}$.

\section{Grupo 2013 metodología tradicional}

Esta metodología consistió en clases expositivas y resolución de ejercicios. El profesor abordó contenidos detectados débiles en curso de nivelación, utilizó como recurso presentaciones y pizarra. No hubo planificación previa por parte de los estudiantes frente a las actividades a realizar.

\section{Grupo 2014 metodología de autorregulación del aprendizaje}

En el grupo de estudiantes 2014, la metodología consideró las tres fases del proceso de autorregulación del aprendizaje señaladas por Schunk y Zimmerman 1994; 2002: planificación, ejecución y reflexión ${ }^{3,4}$.

La planificación de actividades refiere a los procesos que organizan los esfuerzos por aprender. El estudiante ordena la realización de su trabajo frente a la meta determinada por el docente ${ }^{12,13}$. El docente activa en los estudiantes conocimientos previos, vivencias, experiencias, intereses, motivaciones y establece las metas de aprendizajes en forma consensuada.

La ejecución de actividades implica los procesos que tienen lugar durante el aprendizaje y que afectan la atención, la ejecución del trabajo y la memorización posterior. En esta fase, el docente explica el sentido y significado que tiene para el estudiante la tarea, sus proyecciones, su utilidad, la claridad y precisión de lo que se está exigiendo y lo que se espera que aprenda ${ }^{14,15}$.
El docente estimula en el estudiante diferentes niveles de comprensión, mediante ejercicios altamente contextualizados a la disciplina y a la vida diaria. Las actividades presentan diferentes grados de dificultad, estimulando el recuerdo de información, la interpretación de datos, el análisis y aplicación de la información, la evaluación y resolución de problemas.

La fase final de reflexión se relaciona con el cierre del aprendizaje. Esta fase considera los procesos que tienen lugar tras el esfuerzo por aprender y que influyen en la organización y retención de lo aprendido. Implica para el estudiante tomar conciencia sobre las expectativas acerca del trabajo realizado ${ }^{14,16}$. El docente evalúa con los estudiantes el logro de los objetivos, corrige y retroalimenta lo alcanzado. Se insta a que tomen conciencia del porqué de los errores y se les invita a comunicar las soluciones que podrían mejorar su eficiencia en la resolución de los problemas trabajados.

La diferencia del trabajo realizado en ambas metodologías se muestra en la Tabla 1.

\section{Capacitación docente}

Durante el segundo semestre de 2013, los profesores fueron invitados a participar de un curso de perfeccionamiento docente dictado por la Oficina de Educación Médica. Este curso se desarrolló durante los meses de junio a diciembre y tuvo una duración de 100 h pedagógicas. El curso se enfocó en conducción de clases y metodologías docentes. Utilizó como estrategia metodológica el enfoque de autorregulación del aprendizaje, con cada uno de los componentes alineados a los momentos de una clase y en las actividades desarrolladas (Tabla 1).

Para evaluar el impacto de las metodologías utilizadas sobre el rendimiento académico, se compararon los resultados logrados por los estudiantes de ambos grupos considerando:

- Calificaciones obtenidas en las asignaturas Biología celular y Bioquímica.

- Número de aprobaciones y reprobaciones cursadas en ambos grupos.

- Para el análisis inferencial de los datos se utilizó Graph Pad Prism, se consideró un nivel de confianza de $95 \%$ y un error de $5 \%$.

- Test t-Student para diferencias de medias entre variables continuas (variables de ingreso y calificaciones). 
Tabla 1. Comparación de las metodologías utilizadas en los cursos de Biología celular y Bioquímica en la carrera de Medicina (Grupo 2013 y 2014)

\begin{tabular}{|c|c|c|}
\hline $\begin{array}{l}\text { Momentos } \\
\text { de la clase }\end{array}$ & $\begin{array}{l}\text { Metodología Tradicional } \\
\text { Grupo } 2013\end{array}$ & $\begin{array}{l}\text { Metodología Autorregulación } \\
\text { Grupo } 2014\end{array}$ \\
\hline \multirow{8}{*}{$\begin{array}{l}\text { Inicio de la } \\
\text { clase }\end{array}$} & \multicolumn{2}{|l|}{ Planificación de actividades } \\
\hline & \multirow{3}{*}{$\begin{array}{l}\text { Presentación del profesor } \\
\text { Nombre y asignatura } \\
\star * \star * *\end{array}$} & Presentación del profesor \\
\hline & & Nombre y asignatura Intencionalidad, objetivos, metas \\
\hline & & Aplicación tópico generador \\
\hline & \multirow{2}{*}{$\begin{array}{l}\text { Exposisicón del tema } \\
\star \star \star \star \star\end{array}$} & Planificación de las actividades \\
\hline & & Ordenamiento de las actividades y sentido \\
\hline & $\begin{array}{l}* * * * * \\
* * * * *\end{array}$ & Organización de respuestas \\
\hline & $* * * * *$ & Sentido de autoeficacia: Cómo alcanzar las metas \\
\hline \multirow{7}{*}{$\begin{array}{l}\text { Desarrollo de } \\
\text { la clase }\end{array}$} & \multicolumn{2}{|l|}{ Ejecución de actividades } \\
\hline & \multirow{4}{*}{$\begin{array}{l}\text { Exposisición conceptos fundamentales } \\
\text { Demostración ejercicios en pizarra } \\
\star * * * * * \\
\star * * * * *\end{array}$} & Entrega de instrucciones \\
\hline & & Aportes de conceptos fundamentales \\
\hline & & $\begin{array}{l}\text { Trabajo con actividades de recuerdo, inferencia, evaluación y } \\
\text { solución de problemas }\end{array}$ \\
\hline & & Trabajan con actividades altamente contextualizadas \\
\hline & $* * * * *$ & Indagan y buscan información complementaria \\
\hline & $* * * * *$ & Registran y resuelven dudas, ejemplifican \\
\hline \multirow{7}{*}{$\begin{array}{l}\text { Cierre de } \\
\text { actividades }\end{array}$} & \multicolumn{2}{|l|}{ Reflexión } \\
\hline & Revisan y corrigen ejercicios & Responden tópico generador inicial \\
\hline & \multirow{2}{*}{$\begin{array}{l}\text { Resuelven ejercicios como tareas } \\
* * * * *\end{array}$} & Reflexionan sobre la importancia de lo aprendido \\
\hline & & Reconocen fortalezas y debilidades durante la ejecución \\
\hline & $* * * * *$ & Generan nuevas interrogantes \\
\hline & $* * * * *$ & Detectan vacíos de información \\
\hline & $* * * * *$ & Completan la información como trabajo independiente \\
\hline
\end{tabular}

- Test exacto de Fisher como medida de asociación entre metodología docente y reprobación.

- Se calcula Odds Ratio como coeficiente de probabilidad de reprobar o aprobar las asignaturas.

\section{Resultados}

Nuestro análisis de equivalencia demostró que ambos grupos de estudiantes eran comparables en variables de admisión, conocimientos científicos iniciales, comprensión lectora y estrategias de estudios reconocidas mediante el test de Schmeck ${ }^{11}$ (Tablas 2, 3 y 4 ).

El impacto de las metodologías utilizadas en el curso de nivelación demostró que en ambos grupos los porcentajes de aprobación aumentaron. De 13\% de aprobación inicial aumentó a 64\% en los estudiantes 2013 ( $\mathrm{p}<0,0001$ ). Para el grupo 2014 , de $22 \%$ de aprobación inicial aumentó a $96 \%$ de aprobación final $(\mathrm{p}<0,0001)$ (Figura 2). Sin embargo, a pesar de obtener avances importantes en ambas metodologías, se observó que el grupo 2014 enfrentados a una metodología con autorregulación del aprendizaje obtuvieron en promedio, mejores calificaciones finales con menores dispersiones entre ellas (Tabla 5). Al analizar las diferencias absolutas entre la nota obtenida en el pre test y la nota obtenida en el post test de cada alumno se obtuvieron diferencias significativas a favor del grupo con metodología autorregulada $(\mathrm{p}=0,0017)$.

Al comparar las calificaciones finales (resultantes de tres pruebas solemnes y un examen) de las asignaturas de Biología celular y Bioquímica logradas por ambos grupos, se observaron diferencias en promedios y rangos a favor del grupo 
Impacto de una metodología de autorregulación del aprendizaje en medicina - C. Williams et al

Tabla 2. Distribución estadística de la muestra según las variables intervinientes: edad, sexo y establecimiento escolar de procedencia

\begin{tabular}{|lllll|}
\hline Criterios & & Grupo 2013 & Grupo 2014 & Valor p \\
\hline Sexo & $\mathrm{n}$ & 85 & 85 & 0,511 \\
\hline Edad & Mujeres (\%) & $48(56 \%)$ & $40(47 \%)$ & 0,053 \\
\hline NEM & Media \pm DS & $19,08 \pm 0,79$ & $18,7 \pm 0,66$ & 0,8725 \\
\hline PSU & Media \pm DS & $6,17 \pm 0,32$ & $6,26 \pm 0,32$ & 0,2607 \\
Ciencias & Media \pm DS & $689,1 \pm 23,96$ & $698,9 \pm 18,21$ & 0,4664 \\
\hline Municipalizados & Media \pm DS & $672,6 \pm 51,86$ & $685.4 \pm 48,65$ & 0,3561 \\
\hline Subvencionados & $\mathrm{n}(\%)$ & $11(13 \%)$ & $7(8 \%)$ & $25(29 \%)$ \\
\hline Particulares & $\mathrm{n}(\%)$ & $26(31 \%)$ & $53(62 \%)$ & \\
\hline
\end{tabular}

Tabla 3. Frecuencia absoluta de la muestra en rangos PSU (Lenguaje/Matemáticas) y PSU Ciencias 2013-2014

\begin{tabular}{|lcc|}
\hline PSU Leng/Mat & $\mathbf{2 0 1 3}$ & $\mathbf{2 0 1 4}$ \\
\hline $630-650$ & 3 & 1 \\
$651-699$ & 43 & 48 \\
700 o $>$ & 39 & 36 \\
\hline Promedio & 689,6 & 698,9 \\
\hline
\end{tabular}

Valor p 0,2607*

\begin{tabular}{|lcc|}
\hline PSU Ciencias & $\mathbf{2 0 1 3}$ & $\mathbf{2 0 1 4}$ \\
\hline $630-650$ & 10 & 14 \\
$651-699$ & 59 & 48 \\
700 o $>$ & 16 & 23 \\
\hline Promedio & 672,6 & 685,4 \\
\hline
\end{tabular}

Valor p 0,4664*.

Tabla 4. Comparación estadística de grupos 2013 y 2014, según resultado del test de comunicación efectiva (comprensión y estrategia de estudio, realizado por la Universidad Finis Terrae)

\begin{tabular}{|llc|}
\hline Comparación ambos grupos t-Student & & \multicolumn{2}{c|}{$\begin{array}{c}\text { Val3 vs } 2014 \\
\text { Valor p }\end{array}$} \\
\hline Test comunicación efectiva, comprensión de textos & Descripción explícita & 1,892 \\
& Descripcion Inferencial & 2,643 \\
& Valor p, promedio test & 0,8366 \\
Estrategia de estudios, test de Schmeck & Nota promedio del grupo & 5,8 \\
Dimensiones de estilos de aprendizajes, test de Schmeck & Estudio metódico & 0,1790 \\
& Estilo profundo & 1,000 \\
& Estilo superficial & 0,6313 \\
\hline
\end{tabular}

Test informal de comunicación efectiva (Vicerrectoría Académica Universidad Finis Terrae) y Test de Schmeck

autorregulado, siendo estas diferencias significativas tanto para la asignatura de Biología celular como de Bioquímica, ambas dictadas en el primer semestre de la carrera $\left(\mathrm{p}=0,0341^{\star}\right.$ y $\left.0,0323^{\star}\right)$ (Tabla 6).
Finalmente, al analizar los porcentajes de reprobaciones obtenidas en cada grupo por asignaturas, se observó una disminución de alumnos reprobados en las asignaturas que trabajaron con metodología de autorregulación (Figura 3). 


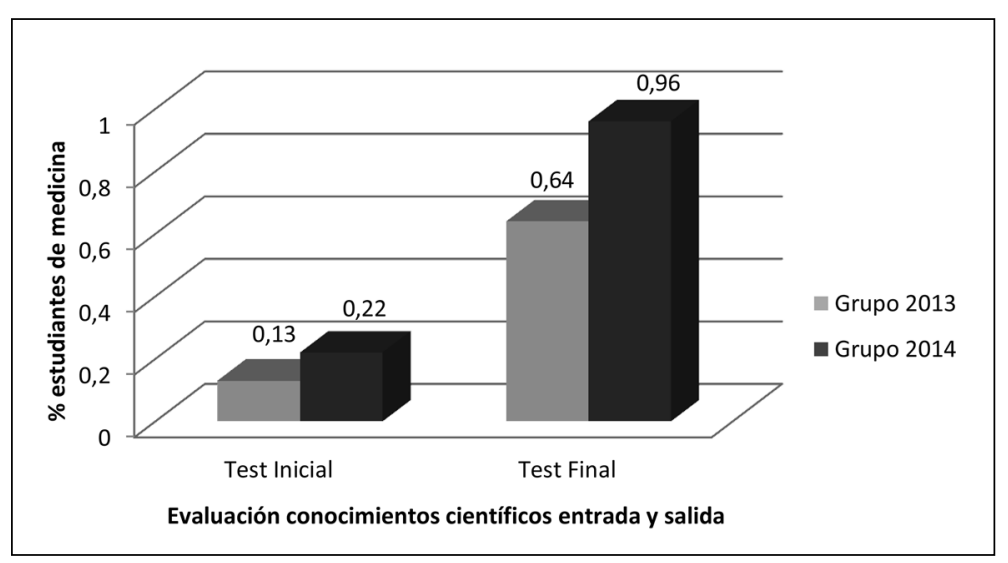

Figura 2. Porcentaje de estudiantes que obtienen notas igual o superior a 4,0 en test inicial y final.

Tabla 5. Rendimiento entre pre test y post test de conocimientos científicos en Grupo 2013 y 2014

\begin{tabular}{|lc|c|c|}
\hline Grupos de estudio & $\begin{array}{c}\text { Nota test inicial } \\
\text { Media } \pm \text { SD }\end{array}$ & $\begin{array}{c}\text { Nota test final } \\
\text { Media } \pm \text { SD }\end{array}$ & $\begin{array}{c}\text { t-Student } \\
\text { Valor p }\end{array}$ \\
\hline 2013 Tradicional & $3,1 \pm 0,73$ & $4,2 \pm 0,83$ & $<0,0001$ \\
2014 Autorregulación & $3,3 \pm 0,86$ & $5,0 \pm 0,63$ & $<0,0001$ \\
\hline Valor $p$ & $>0,1087 \quad \nabla$ & $<0,0006 \quad \downarrow$ & \\
\hline
\end{tabular}

Tabla 6. Rendimiento notas finales de asignaturas Biología celular y Bioquímica en Grupo 2013 y 2014

\begin{tabular}{|c|c|c|c|c|}
\hline \multirow[t]{2}{*}{ Cohorte Estudio } & \multicolumn{2}{|c|}{ Biología celular } & \multicolumn{2}{|c|}{ Bioquímica } \\
\hline & $\begin{array}{l}\text { Rangos de nota } \\
\text { mínima y máxima }\end{array}$ & $\begin{array}{c}\text { Promedio } \\
\text { de nota curso }\end{array}$ & $\begin{array}{l}\text { Rangos de nota } \\
\text { mínima y máxima }\end{array}$ & $\begin{array}{c}\text { Promedio } \\
\text { de nota curso }\end{array}$ \\
\hline Grupo 2013 & $1,3-5,6$ & 4,4 & $1,2-6,2$ & 4,6 \\
\hline Grupo 2014 & $3,5-6,6$ & 5,0 & $3,1-6,6$ & 5,3 \\
\hline Valor $p$ & \multicolumn{2}{|c|}{$0,0341 *$} & \multicolumn{2}{|c|}{$0,0323^{*}$} \\
\hline
\end{tabular}

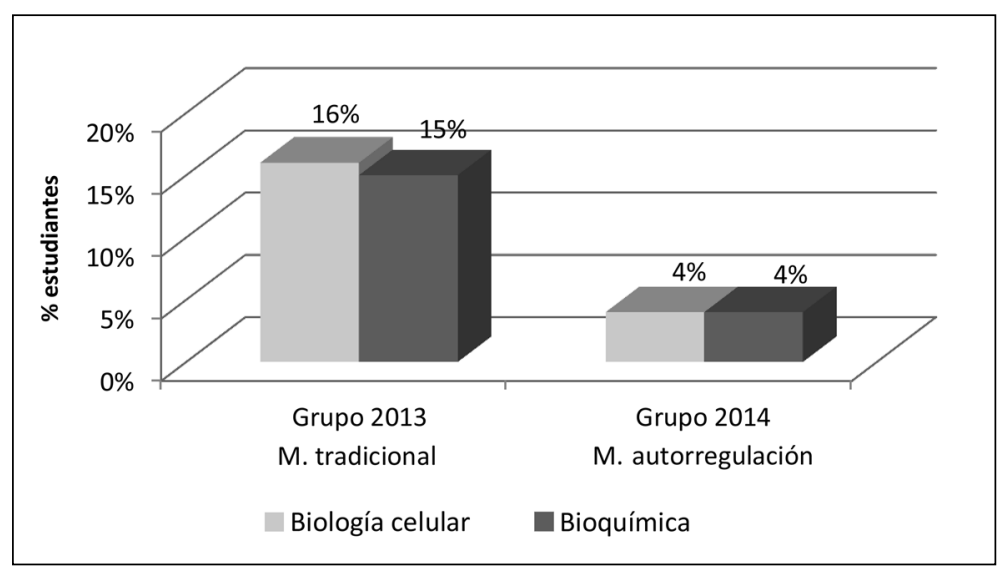

Figura 3. Porcentaje de estudiantes que reprueban las asignaturas de Biología celular y Bioquímica en 2013 y 2014. 


\section{Tabla 7. Resultados test exacto de Fisher y cálculo de Odds ratio (OR) como medida de efecto}

\begin{tabular}{|llcc|}
\hline Asignatura & Fisher & OR & Cl \\
\hline Biología celular & $0,0081^{* *}$ & 0,25 & 0,07 a 0,68 \\
Bioquímica & $0,01^{*}$ & 0,27 & 0,07 a 0,73 \\
\hline
\end{tabular}

Al aplicar el test exacto de Fisher entre metodología y número de reprobaciones, se obtienen valores significativos a favor de la metodología con autorregulación (Biología celular $\mathrm{p}=0,02 \mathrm{y}$ Bioquímica $\mathrm{p}=0,03$ ).

El cálculo de Odds ratio (OR), nos indica que la razón entre reprobar y aprobar las asignaturas de Biología celular fue de 0,25 veces y Bioquímica de 0,27 veces. Lo que significa que la probabilidad de reprobar la asignatura fue menor en estudiantes que trabajaron la metodología de autorregulación en comparación a estudiantes que trabajaron la metodología tradicional (Tabla 7).

\section{Discusión}

El análisis de equivalencia realizado demostró que al inicio de las actividades académicas ambos grupos presentan deficiencias similares en conocimientos científicos, en estrategias de estudio y procesamiento de información. Deficiencias también detectadas en otros estudios similares de educación superior ${ }^{8,17,18}$.

El análisis del programa de nivelación reveló que en ambos grupos existió un aumento significativo de estudiantes que aprueban el post-test, demostrando que los programas de nivelación logran enseñar los conocimientos científicos necesarios para las asignaturas del primer semestre, independiente de la metodología utilizada. Esta situación ha sido documentada por diversos autores ${ }^{9,19}$.

Sin embargo, se observa que los estudiantes que trabajan la metodología de autorregulación obtienen mejores resultados académicos, por lo que, la incorporación de esta metodología permite adquirir un mejor dominio del conocimiento científico.

Al observar las calificaciones logradas al finalizar las asignaturas de Biología celular y Bioquímica, la metodología con enfoque de autorregu- lación que se utilizó con el grupo 2014 muestra mejores calificaciones y mejores porcentajes de aprobación. Esto último es muy coincidente con lo que indica la literatura, la que señala que los estudiantes tienen mayor control sobre sus propios procesos de aprendizaje, mejorando su rendimiento académico ${ }^{5,12,14,15}$. Por lo que, el profesor debe facilitar y guiar al estudiante para planificar, ejecutar y reflexionar su propio proceso de aprendizaje, de modo que el estudiante pueda desempeñarse exitosamente en las áreas de su disciplina ${ }^{16}$.

Si bien los resultados de este estudio demuestran una mejoría en el rendimiento de los estudiantes con metodología de autorregulación, no permiten discriminar si este impacto es producto de cada una de las etapas o corresponde a la sumatoria de todas las fases del proceso de autorregulación, tales como, planificación de su trabajo académico, ejecución o reflexión. Lo anterior muestra la necesidad de evaluar en forma diferida el impacto de cada uno de estos componentes.

Actualmente, se ha podido constatar que todos aquellos docentes que están incorporando la metodología de autorregulación del aprendizaje en sus clases, disminuyen considerablemente las tasas de reprobación en sus asignaturas. En el caso particular de Biología celular y Bioquímica, en las cuales esta metodología se incorpora en el año 2014, los porcentajes de reprobación disminuyen desde $15 \%$ en el 2013 a 3\% en la actualidad (datos no publicados). Estos resultados validan que la incorporación de este tipo de metodología mejora el proceso de enseñanza-aprendizaje de los estudiantes.

Finalmente, surge la necesidad de abordar este estudio de manera cualitativa, con el fin de determinar percepciones de los docentes y de los estudiantes con respecto a la metodología de autorregulación.

\section{Conclusiones}

La metodología de autorregulación del aprendizaje mejora el rendimiento académico en los estudiantes de medicina de primer año en las asignaturas de Biología celular y Bioquímica, impactando de manera positiva en las tasas de aprobación y fortaleciendo a través del proceso de enseñanza-aprendizaje la adquisición de conocimiento científico. 


\section{Referencias}

1. Escorcia R, Gutiérrez A, Hermes de Jesús A. La educación superior frente a las tendencias sociales del contexto. Educación y Educadores 2007; 10 (1): 63-77.

2. Pintrich PR. The role of goal orientation in self-regulated learning. En: M. Boekaerts, P.R. Pintrich y M. Zeidner, Editores, Handbook of self-regulation. San Diego, California: Academic Press.; 2000. p. 452-502.

3. Schunk D, Zimmerman B. Self-Regulation in Education: Retrospect and prospect. En: Schunky D, Zimmerman BJ. Editores, Self-regulation of learning and performance: Issues and educational applications. Hillsdale, NJ: Lawrence Erlbaum Associates, Inc.; 1994. p. 305-314.

4. Zimmerman BJ. Attainment of self-regulation: A social cognitive perspective. In M. Boekaerts P.R. Pintrich y M. Zeidner Eds, Handbook of self-regulation. San Diego, CA: Academic Press.; 2002. p.13-39.

5. Núñez J, Solano P, González-Pienda J, Rosario P. El aprendizaje autorregulado como medio y meta de la educación. Papeles del Psicólogo 2006 b; 21 (2): 143-76.

6. Elvira-Valdés MA, Pujol L. Autorregulación y rendimiento académico en la transición secundaria-universidad. Revista Latinoamericana de Ciencias Sociales, Niñez y Juventud 2012; 10 (1): 367-78.

7. Hernández F, Salas P, Cuesta J. Impacto de un programa de autorregulación en el aprendizaje en estudiantes de grado. Revista de Educación 2010; 353: 571-88.

8. Carbonero M, Navarro J. Entrenamiento de alumnos de Educación Superior en estrategias de aprendizajes en matemáticas. Psicotherma 2006; 18 (3): 348-52.

9. Santelices L, Williams C, Soto M, Dougnac A. Efecto del enfoque de autorregulación del aprendizaje en la enseñanza de conceptos científicos en estudiantes universitarios en ciencias de la Salud. Rev Med Chile 2014; 142 (3): 375-81.

10. Santelices L, Williams C, Zárate A, Soto M, Jara N, Dougnac A. Impacto de un programa de nivelación de ciencias básicas en estudiantes de primer año de la carrera de medicina. Rev Med Chile 2013; 141 (6): 710-5.

11. Schmeck RR, Ribich F. Construt validation of the Inventory of Learning Processes. Applied Psychological Measurement 1998; 2: 551-62.

12. De la Fuente J, Lozano A. Assessing selft-regulated learning in early childhood education: Difficulties, needs and prospects. Psicotherma 2010; 22 (2): 278-83.

13. Garello MV, Rinaudo MC. Autorregulación del aprendizaje, feedback y transferencia de conocimiento: Investigación de diseño con estudiantes universitarios. REDIE 2013; 15 (2): 131-47.

14. García Martín M. La autorregulación académica como variable explicativa de los procesos de aprendizaje universitario. Profesorado. Revista de currículum y Formación del profesorado 2012; 16 (1): 203-22.

15. De la Fuente J, Pichardo MC, Justicia F, Berbén A. Enfoques de aprendizaje, autorregulación y rendimiento en tres universidades europeas. Psicothema 2008; 20 (4): 705-11.

16. García T, Pintrich PR. Regulating motivation and cognition in the classroom: The role of self-schemas and self-regulatory strategies. En Schunk DH, Zimmerman BJ. Editores, Self-regulation of learning and performance. Issues and educational applications. Hillsdale, NJ: Lawrence Erlbaum Associates, Inc.; 1994. p. 320-5.

17. Tejedor FJ, García-Valcárcel A. Causas del bajo rendimiento del estudiante universitario (en opinión de los profesores y alumnos). Propuestas de mejora en el marco del EEES. Revista de Educación 2007; 342: 443-73.

18. Díaz C. Modelo conceptual para la deserción estudiantil universitaria chilena. Estudios Pedagógicos 2008; 34 (2): 65-86.

19. Dapelo B, Matus M. Necesidades de fortalecimiento cognitivo motivacional en estudiantes universitarios de primer año: Implicaciones para una nivelación efectiva de competencias. Revista de Orientación Educacional 2013; 27 (52): 15-33. 